\title{
Contextualize of Disaster Nursing Competencies within Resilient Health Care System in Saudi Arabia
}

\author{
Banajah S* \\ King Saud University, Saudi Arabia
}

*Corresponding author: Samah Banajah, King Saud University, Saudi Arabia, Tel: 00966559445327; Email: sbanajah@ksu.edu.sa

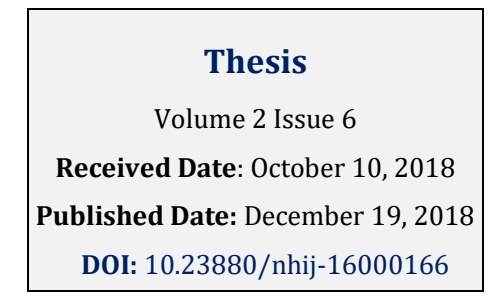

Keywords: Nursing; Healthcare; Epidemiology

Abbreviations: COE: Centre of Excellence; ICN: International Council of Nurses; NEPEC: Nursing Emergency Preparedness Education Coalition; CRED: Centre for Research on the Epidemiology of Disasters; UNISDR: United Nation International Strategy for Disaster Reduction; WHO: World Health Organization; ICS: Incident Command System; PPRR: Prevention, Preparedness, Response and Recovery; QUB: Queens University Belfast.

\section{Introduction}

\section{Background}

Globally, around 6,457 weather-related disasters such as earthquakes, floods, cyclones and landslides claimed 606,000 lives and affected more than 4 billion people between 1995 and 2015, according to an international disaster data base. According to the Centre for Research on the Epidemiology of Disasters (CRED), According to the Centre for Research on the Epidemiology of Disasters, since 2015, when 346 weather-related disasters were reported, these figures have been increasing, with approximately 205 million people now affected each year [1].

Tragically, the statistic extremely elevated in 2017, in which catastrophic natural losses were 93 percent higher than the 2000-2016 average. These statistics do not include casualties arising from non-natural, human-made disasters such as war, stampedes, terrorism, transport accidents and even infectious outbreaks.
There are various ways of understanding what is meant by a disaster. Guidance issued by the World Health Organization [2], defines the term as 'A widespread destruction of the environment, the economic, social and healthcare infrastructure, as well as loss of life, overwhelming the ability of individuals and the community to respond using their own resources' [2]. A similar description is offered by the United Nations in its Strategic Guidance for Disaster Reduction, in which disaster is defined as a 'serious disruption of the functioning of a community or society involving widespread human, material, economic or environmental losses and impacts, which exceeds the ability of the affected community or society to cope using its own resources' [2].

Saudi Arabia has experienced many catastrophic events, particularly flooding, in the west of the country. Recently, a mass-casualties incident involving a fire at the Jezan hospital in the south of the Kingdom which resulted in the deaths of more than 25 patients and 175 injured casualties was reported in social media. Such events demonstrate the inadequacies of the healthcare system in the Kingdom when responding to such disasters, with a particular problem being the lack of healthcare workers with disaster competencies within a national disaster response team. Given that the World Health Organization has declared that no healthcare system should be considered prepared unless its nurses are prepared [2]. The emergency nurses in Saudi Arabia perceive themselves as unready to respond effectively to disasters [3].

The current inadequacies of the Saudi healthcare system in terms of disaster response reflect the inadequacy of disaster training for healthcare workers, 


\section{Nursing \& Healthcare International Journal}

which itself has resulted in a lack of competency within these professions [4]. Therefore, there is an urgent need to develop disaster- competent healthcare personnel by adopting standard disaster operation competencies against which the readiness of Saudi healthcare workers can be assessed.

This need is not unique to Saudi Arabia. Despite the devastating impact of natural disasters, the response is still below standard in many countries. For example, the United Nations declared its dissatisfaction with the standard disaster response to the Haiti Earthquake [5].

Countries around the world invest considerable resources in preparing to respond effectively to disasters. Because of their diverse knowledge and experience, nurses are well-equipped to serve on the frontline in any disaster. However, the nature of disasters requires frontline healthcare workers to possess additional competencies to ensure an effective, efficient and coordinated response. Integrating disaster competencies into nursing education and training will prepare nurses to deliver an effective response in the event of a disaster.

An effective response must be associated with effective management of health care resources. In most cases, the most viable resources are trained disaster nurses who are capable of providing standard care in all unexpected conditions and therefore are able to support the response of the health care system. International council of nursing supporting this by providing the guiding to prepare the nurses with disaster competencies competent disaster nurse would be the most valuable resource in disaster response [2].

That said, while nurses demonstrate a high level of competence in emergency triage, disaster triage requires a much higher level of judgment and advanced decisionmaking skills in order to priorities causalities appropriately, that is on the basis of who will gain the most benefit from limited resources. Efficient disaster triage depends on the ability of nurses to work within a very basic range of interventions and decide which patients will benefit most. Therefore, if they are to serve as frontline respondents in a disaster, emergency nurses must enhance their disaster triage skills by mastering the competencies that Anderson (2012) [6] considered being essential.

Typically, following a disaster, nurses must meet the multiple care needs of large numbers of victims in a chaotic environment with limited, if any, resources. Even in the most difficult circumstances, however, the principle of standard care must not be compromised. Cusack, et al. (2015) [7] Proposed that "adapted standard care" be applied when delivering care in a disturbed environment. Disaster victims have a wide range of care needs that often exceed the available resources in the immediate aftermath. Disaster nurses care for disaster victims in unusual conditions and yet are expected to deliver the same high standard of care that is associated with the nursing profession.

Ensuring competent healthcare workers are available is vital to the delivery of an effective disaster response. Many education and training programs have been developed by a wide range of institutions and organizations to prepare nurses to respond to disasters, however, the competencies were neither consistent nor evidenced-based until 2009 [8], when the International Council of Nurses' established its Framework of Disaster Nursing Competencies [2]. This framework was followed by the development of national standardized "all-hazard" disaster core competencies for all other healthcare workers in the United States [9].

The process for developing nursing competencies in disaster response takes many forms, from generating a set of competencies specifically for healthcare workers with responsibility for responding to disasters to establishing a hierarchy of skills, from basic to advanced, which healthcare workers acquire consecutively [8]. To achieve consensus, competency should be based on the opinion of experts in disaster management and validated by evidence of best practice [8].

\section{Knowledge Gap}

As previously noted, emergency nurses in Saudi Arabia perceive themselves as unready to respond effectively to disasters [3]. The media have reported evidence that poor management in the Saudi health care system reduces effectiveness during disasters, especially when the number of victims exceeds the capabilities of nurses to deal with cases due to lack of experience with masscasualty incidents. There is therefore an urgent need to enhance the effectiveness of disaster response qualifications, particularly among nurses in Saudi Arabia.

Saudi Arabia is moving the emergency response of its health care system toward prevention and preparedness for disaster, and nurses are considered the most viable healthcare workers because they have already been trained to be first responders in the event of such disasters [4]. 


\section{Nursing \& Healthcare International Journal}

\section{Aim of the Study}

The aims of this study are to analyze the existing templates for assessing the knowledge and skills of emergency nurses and then to up-skill each level with a suggested set of competencies. Towards this end, the current levels of disaster response competencies will be determined by identifying the standard disaster competencies that disaster respondents are required to develop and assessing whether this set of competencies is valuable and appropriate for up skilling practitioners to the level of competency required of disaster respondents. The findings from this research will form the basis for recommendations regarding the introduction, implementation and delivery of further competencies in the future.

Up-skilling emergency nurses to ensure an effective response to disasters has been proposed as part of the ICN framework for developing standard disaster competencies. Its aim is to equip emergency nurses with disaster response competencies to be a competent healthcare professional in responding to a disaster.

Each set of competencies was assigned to the stage of nursing experience. Clustering the domain of competencies into a hierarchy based on education and acquired expertise. The skills building process will begin with integrative core disaster competencies aimed at the nursing group at the base of this hierarchy and become progressively more advanced. Nurses at the peak of their profession therefore will have attained the advanced disaster competencies set. This approach will provide an organizing structure for the position of nurses aligned with their level of experience. In other words, nurses in positions of higher authority will be expected to demonstrate advanced disaster competencies.

While many disaster competencies have been developed to prepare nurses to respond to disasters, these competencies lack consistency, nor are they evidenced base [8].

Analyzing the current existing knowledge and skills for emergency nurses related to designed templates and then up-skilling each level with suggested set of competencies. Planning to find the current existing levels of disaster response competencies by identifying the developed competencies that required for disaster respondents adopting standard developed disaster competencies. Find whether these set of competencies are valuable and appropriate to enhance and up-skilling their level to be competent disaster respondents. Then introducing these competencies up to Implementing to be further for delivering in the future recommendations.

\section{Research Questions}

To what extent are emergency nurses in Saudi Arabia prepared in relation to recognized international standards of preparedness in relation to managing disasters?

\section{Objectives}

- To measure the current competency level (knowledge and skills) of registered emergency department nurses in Saudi Arabia in order to evaluate and critique their preparedness to respond to disasters.

- To determine level of disaster competency level among emergency nurses.

- To discuss the views of stakeholders regarding the disaster nursing response within resilient disaster field. (A disaster field is a designated area allocated during the disaster).

- To understand the mechanisms that interferes with and facilitates the role of nurses within the Saudi disaster response system.

- To propose an appropriate education strategy for upskilling the competence of emergency nurses in Saudi Arabia.

\section{Theoretical Framework}

The proposed discussion of disaster response nursing within the Saudi health care system is based on two integrated scope. The first involves defining the competency standards for education and training in order to prepare nurses for disaster response. The second involves developing preparedness measures designed to reduce the impact of disaster for a more efficient response. This is achieved by reducing the risk of disaster through a detailed examination of the United Nations' International Disaster Standards Framework for preparing the health workforce as one measure of effectiveness in reducing disaster risks. In keeping with the aim of this study, the focus is on nursing competencies in preparedness and preparedness phases of disaster management in order to build the nursing capacity within a resilient, responsive health care system.

The national response of Saudi Arabia in moving toward the development of a resilient heath care system is part of its commitment to the United Nation International Strategy for Disaster Reduction (UNISDR) 


\section{Nursing \& Healthcare International Journal}

known as the Disaster Risk Reduction Framework for Building a Resilient Health Care System [10]. Drawing on relevant legislation from around the world, the UN and the WHO established a global standard for disaster management in 2005 which sought chiefly to reduce the risk of disaster by targeting the disaster preparedness of national health care systems through enhanced training of their workforces.

Recognizing that health care systems must have adaptive capabilities, given the inherently unexpected nature of disaster, this framework emphasizes enhancing preparedness activities of these systems and improving the competencies of the health care workforce through education and training, not only within the health institutions themselves, but through further collaboration with all responding organizations.

In terms of its approach to disaster management, the world has shifted to reducing the risk of disaster rather than responding to events (according to the United Nation International strategies in 2015) [10]. In keeping with this strategy, Priority 4 of the Sendai Frame work involves enhancing the preparedness of nurses as well as other health workers. The Sendai Framework [10] set the standard for lowering the risk and hazards of disaster of particular relevance to this research is its recognition that risk reduction is a shared responsibility, and its emphasis on training health care professionals to ensure their ability to respond effectively and the vital importance of coordination and cooperation among responding teams, sharing information about best practices and the mutual allocation of resources during all phases of disaster management [10]. Saudi Arabia and other 167 states have committed to adhere to the United Nations action plan for reducing disaster risks. Therefore, preparing the workforce for their role in disaster risk reduction is crucial to ensure an effective health care response. This framework provides a structure for preparedness activities that emphasize enhancing the training of health care workforce in order to achieve the best response. Additionally, it coordinates the mechanisms within health care institutions, between these institutions and the health care system, and between the system and the government. These disaster preparedness activities include establishing a safe disaster field, improving communication within the assigned response team, and maintaining an up-to-date disaster response progress registration database.

This disaster reduction framework (UNISDR) also has been chosen because it emphasizes stakeholder engagement and the importance of their close cooperation with responding workforces. The framework provides international standards for developing disaster preparedness plans and strategies for delivering effective response systems based on the mechanisms between stakeholders and their workforce [1].

Improving the disaster competencies among workforce, especially nurses, is one of the priorities for demonstrating compliance with the policies outlined in the United Nations' international standards for disaster preparedness. This involves up-skilling nurses to disaster nurse status based on their understanding of disaster system standards and their interaction policies.

In response to these international standards for disaster preparedness, the International Council of Nursing (ICN) developed a disaster nursing competencies framework which is considered the foundation for disaster standards generally, and especially for nurses. The ICN collaborated with World Health Organization (WHO) in 2009 to establish the basic knowledge and skills required by those nurses involved in disaster management [2].

To understand the mechanisms within disaster health care system Bowling [11] explained that the dynamics of any such system should be analysed in terms of its structure, process and outcomes. Every disaster health care system has an organizational structure and a chain of command. This concept can be operationalized to an Incident Command System (ICS). An incident command plan provides the basis for a smooth and coherent response to disasters; as such its success relies on the extent to which the responders are confident in their ability to follow the Incident Command System, which provides strategic guidance on all aspects of the response process and defines the role and responsibilities of each responder. In the hospital setting, this plan is known as the Hospital Emergency Command System.

Competency is a type of process within this system. It has been defined by the ICN, 2009 [2] as 'a level of performance demonstrating the effective application of knowledge, skill and judgment'. For nurses, competency can be described as the ability to demonstrate a high level of practice gained through valuable experience that builds on their education. Disaster competencies are demonstrated by the extent of education and training acquired by nurses within one or more disaster response systems. Having a clear and consistent health care system command structure as well as competent nurses both influence the ability of the system to achieve its desired outcome and demonstrate effective preparedness. 
Preparedness is structured into disaster health care systems through the competency training provided to their nurses. These disaster competencies are reflected in the knowledge and skills nurses acquire through their activities within the system, which together ensure effective disaster preparedness (Figure 1).
The International council of nursing in collaboration with World Health Organization establishes the International disaster nursing competencies framework as International consensus guiding the nursing practices during disaster [2].

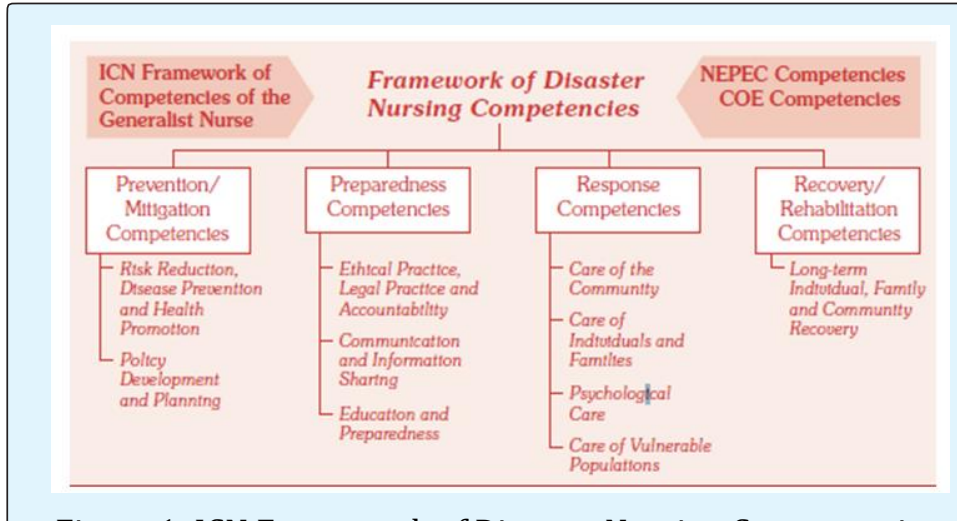

Figure 1: ICN Framework of Disaster Nursing Competencies.

Daily, (2010) [8] highlights un clarity of disaster terminologies required unifying disaster competencies concepts among the respondents with more focus on categorizing the disaster broad areas to more specific the competencies advancing specialized level. Universal United Nation Disaster Reduction as International strategies engaging stakeholders as specialized in disaster response matching each set of required competencies with assigned position i.e competencies may vary depend on nurses' role during disaster [10]. Demonstrating international council of nursing framework for disaster competencies would reveal complete picture of disaster nursing responses based on leveling the disaster competencies in relation to assigned positions [2] (Figure 2).

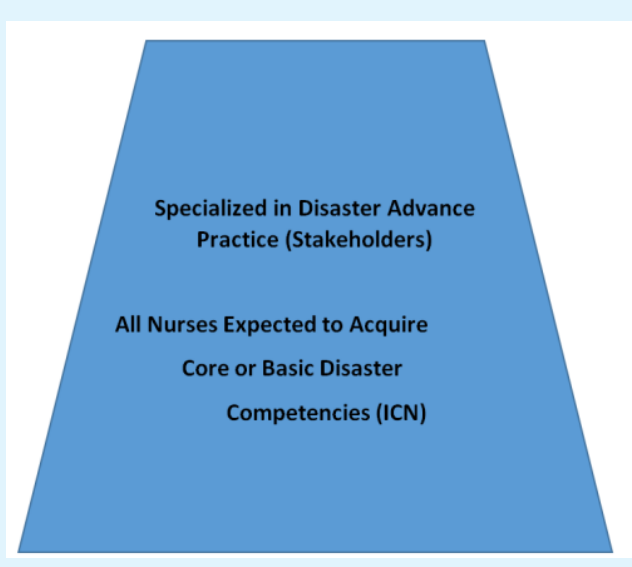

Figure 2: The world leading agency in disaster management.
The United Nation as the world leading agency in disaster management collaborate with World Health Organization to develop the International strategies for disaster reduction plan for 186 countries, Saudi Arabia one of them. These efforts targeting enhancing health care disaster response system to change from responding activities to prevention and preparedness measurement based on change theory toward sustainable development [10].

National response in Saudi Arabia moving toward resilient heath care system as their commitment within United Nation Sendai framework for building resilient health care system. The framework emphasize on enhancing preparedness activities of the health care systems and improving health care workforce competencies through education and training. Not only within the health institutions, but further collaboration with all responding organizations. Unexpected nature of disaster mandates adaptive capabilities from heath care system. Many nurses within health care institution feel unprepared to uncertainty since responding to crisis need expanding their capabilities and transferring their skills in chaotic environment.

Adaptability of the system is the capacity to respond to stresses and shocks. Building the disaster nursing capacity is an integral part of resilient health care system. Resilience is defined as "the capacity of individuals, families, communities, systems and institutions to anticipate, withstand and /or judiciously engage with 
catastrophic events and/or experience". It's challenging for nursing competencies to balance between delivering the high quality for individual victim care within the disaster field. Resilience within health care system measure amount of change in their interventions while maintaining standards of care (Figure 3).

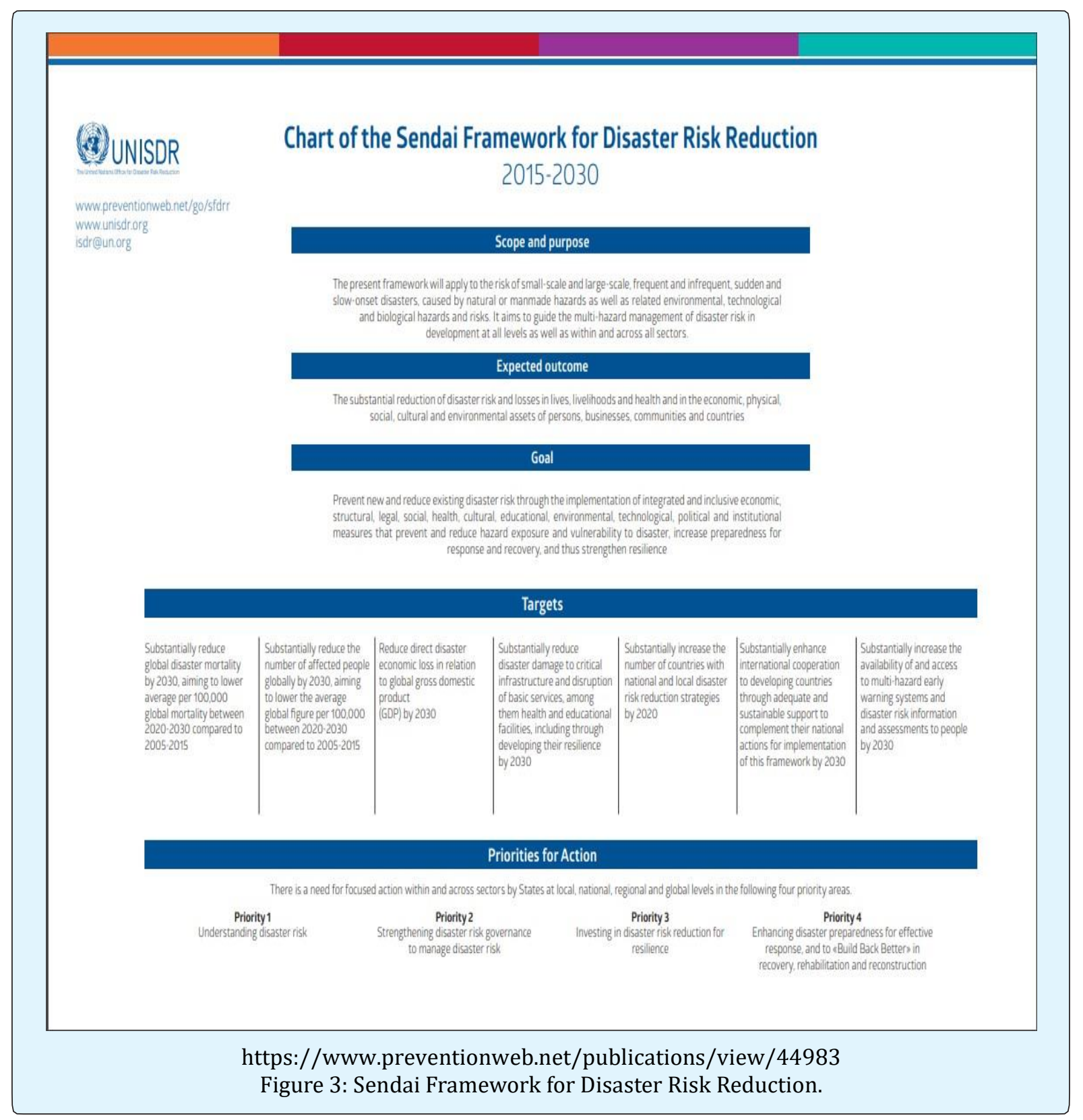

Alraga, (2017) [12] discussing the mechanism of disaster management within health care involve prevention, preparedness, and responding and recovery approach. This was following agreement between World Health Organization with Saudi health care system to structure the Directorate of Health Affairs for disaster management which has authority for all operations with disaster.
This study describing the international strategies of united nation for disaster reduction from nursing prospective. Highlighting the fourth priority of United Nation Framework for disaster reduction through enhancing the disaster preparedness among the healthcare workforces. The education based on internationally agreement disaster competencies healthcare workers preparedness to reach ultimate goal 


\section{Nursing \& Healthcare International Journal}

of reducing the disaster impact and losses as point integrating these two frameworks together.

In Saudi Arabia, floods are considering the most natural disaster frequency at western region. Sand storms are more often in the central and western region. Land slide more frequent in mountainous region in the South of the country. Recurrence of earthquakes in the north with regarding to other natural cause the terrorist attacks distributed over the regions western and south regions are more vulnerable for disaster strikes.

Applying the international strategies emphasize on preparing the disaster responders through knowledge sharing and more awareness with disaster risks. Anticipating the disaster scenario related to recurrent exposure to certain type of disaster in particular area along with understanding four disaster phases consider crucial element in disaster preparedness [10].

\section{Philosophical Assumptions of this Research}

The role process varies with any disaster response mechanisms structure; the role process varies with the command structure Commands. Nurses' roles vary with the system's mechanisms and activities, and these variations affect their level of competency, because the authorities within the system's command structure shape the nurses' responsibilities. In the context of disaster planning, any manipulations or deployment of the roles of nurses will interfere with their level of disaster competency

In 2005, the United Nations, the world's leading agency in disaster management, collaborated with the World Health Organization to develop international strategies for disaster reduction planning for 186 countries, including Saudi Arabia. These efforts focused on enhancing health care disaster response systems to enable the transition from responsive activities to prevention and preparedness measurement based on the theory of change [13].

In 2009, the WHO and the ICN established the International Disaster Nursing Competencies Framework, an expression of international consensus on appropriate guidance for nursing practices during disasters. The study questionnaire has been developed based on the ICN framework to evaluate current levels of disaster competencies among nurses in the Saudi health care system. It has also been used to focus on inducing a change in the approach to preparing nurses to respond to disaster by developing their competencies in line with the United Nations International Strategy for Disaster Reduction (UNISDR).

Alraga's (2017) [12] analysis of the mechanisms of disaster management in the Saudi health care system identified its approach as prevention, preparedness, response and recovery (PPRR). This Disaster health directorate established as following the agreement between the WHO and the Saudi health care system to structure the Directorate of Health Affairs as the primary agent for disaster management with authority for all operations during a disaster. Alraga (2017) [12] Emphasized that the disaster health management structure in the Saudi health care system was based on the disaster continuum model, which consists of all phases (PPRR) of disaster management. Alraga, (2017) recommend harmony between the higher governmental authority the Ministry of Interior and the health care system the Ministry of Health to achieve the ultimate goal of disaster management in accordance with its commitment to international disaster preparedness.

Preparing the health care workforce with disaster competencies is an international strategic priorities for reducing the impact of disasters across the healthcare system. As part of Saudi Arabia's international commitment to disaster preparedness, this study analyses the disaster nursing competencies during the transition from on-site response activities to be competent prepared nurses for health system development which would achieved through this study. The study wills analyses disaster nursing preparedness in order to reduce the impact of disasters in Saudi Arabia and improve the Kingdom's adherence to the UNISDR.

The current disaster management system in the Saudi health care system relies on outdated response activities. Moreover, there is no evidence of preparedness and actual disaster trained nurses are lacking. Also, he highlighted a number of weaknesses in the disaster preparedness plan within Saudi health care system, including inadequate response when disaster strike, improper communication among the responding sectors which affects the coordination of response activities during the disaster and unclear command operations among the responding health care systems.

There was an empirical reflection from disaster response administrators regarding the need to analyses current practice and train disaster response personnel to respond effectively. 


\section{Nursing \& Healthcare International Journal}

Despite its stated commitment to incorporating disaster reduction and prevention, disaster management in the Saudi health system continues to adhere to response activities rather than make the shift to reducing the impact of disaster by ensuring the efficiency and preparedness of health personnel, especially nurses [12].

Strategies developed by competent trained nurses for improving preparedness underscore the urgent need to shift disaster management from response to disaster impact reduction.

Alraga, (2017) [12] discusses incorporating the WHO disaster Health Management Model within the Saudi health care system. The Directorate for Health Affairs for disaster and emergency, which was established as part of that system in 2015, is responsible for setting the legislation that regulates activities during a disaster and for coordinating arrangements between the response sectors as well as the deployment of workforces, including nurses, during disaster to ensure adequate respond [12]. In addition to international strategies for transitioning toward disaster prevention and preparedness, this study emphasizes the need for adherence to international disaster standards of nursing preparedness by the Saudi disaster health management. All these tremendous changes within Saudi health management highlight the need for adequate disaster preparedness among nurses. This can be achieved by integrating disaster competencies into preparedness mechanisms and operations of the Saudi health management system, thereby achieving the international goal of disaster reduction.

\section{Methodology}

Developing an adequate Measure of competency is not a simple task, and there is considerable debate about which methods should be used, especially in relation to nursing. Studies in the UK have tended to favor qualitative methods while quantitative methods are preferred by researchers in the US. Both approaches have been critiqued as inadequate for assessing competency when used alone on the grounds that quantitative methods are "task-focused" while qualitative methods the missing the applicability for different context with quantitative one [14].

Using more than one method to assess the level of disaster competency level produces more reliable results. Moreover, complementing the findings obtained from one method with the results of another enables a deeper understanding of the data. Applying a mixed-method approach supports the data from both types of measurements [15]. In order to convey a complete picture of the level of disaster nursing competency, the evaluation will provide a reasonable explanation of the actual level of competency within the health system.

A Concurrent mixed-methods research design is one in which qualitative data approaches support and are triangulated with quantitative methods, and these approaches are combined throughout the research process. For the purpose of convergent mixed method design of this study, both quantitative and qualitative data will be collected simultaneously [16].

Contextual evaluation considers how the system and its context are interrelated and how their effect on each other explains the outcomes observed [17]. The aim of this evaluation is to explain the mechanism surrounding the current disaster nursing competency level in other words, what works for whom in what circumstances and how.

The study will describe how disaster nursing preparedness for up-skilling supports the disaster response system within a fully resilient health care system. Alternatively, how the disaster response and its international standards regulate the mechanisms surrounding disaster nursing within Saudi context. How the nurses in disaster response roles are regulated by a disaster response system which is contextually influenced. Aligning disaster nursing competencies developed by the ICN with the United Nation international disaster standards for disaster reduction is a common goal. This integration of disaster competencies through the application of the ICN's guidance on disaster mitigation and preparation [2] reflects the importance of disaster competencies among nurses along with the UN's priority of international disaster reduction, which reflects the disaster response in the Saudi health context Preparing nurses in accordance with international standards of disaster competencies is one of the United Nation priorities for reducing the impact of disasters worldwide [1,2].

Disaster nursing activities, competencies, roles, mechanisms and interaction have an effect on and are affected within the Saudi disaster health response, measures to achieve a resilient disaster health care system. This study will describe the disaster response command structure in the health care system from the point of view of stakeholders with responsibility for regulating the role of nurses and guiding their performance. This study will describe the disaster response command structure in the health care system 


\section{Nursing \& Healthcare International Journal}

from the point of view of stakeholders with responsibility for regulating the role of nurses and guiding their performance. A summary of the quantitative and qualitative results from this mixed-methods evaluation will be presented, this methodology is designed to provide a comprehensive evaluation which demonstrates current levels of disaster nursing competency among emergency nurses, explains these outcomes within the context of the Saudi healthcare system and describes the factors that led to these outcomes with the aim of influencing future policy.

\section{Disaster Competencies Measurement}

The issue for the development of disaster nursing in the Kingdom of Saudi Arabia, in line with many other countries, is the lack of a universally accepted tool for assessing disaster nursing competencies Al Thobaity, et al. (2016) [18]. Developed such a tool, which consists scale type questions with psychometric properties to evaluate nurses' competencies in responding to disasters. Al Thobaity, et al. (2016) [18] study demonstrated that this tool is valid and reliable in assessing these competencies and that it might be useful for nursing education in the future. The three core factors the authors identified were competencies in disaster nursing (important for the acute response phase), barriers faced by disaster nursing (crucial for preparedness), and nursing roles in disaster management (important for planning, education and drills). The availability of this instrument is a very recent development, and the authors concluded that it may help to improve disaster preparedness in Saudi Arabia in the future. Therefore, using the most recent valid and reliable instrument will provide a clear picture of the current level of disaster competency among nurses in Saudi Arabia.

\section{Research Design}

\section{Reasons for Conducting Mixed Method Research}

Many factors affect nurses' competency during disaster event responsibility for responding to disaster events and the nature of their role within the responding team. Another factor is the standards governing the response system. Because of the complexity of disaster response within the health care system and an establishing the disaster field with specific criteria and resilient standards. Effective disaster management requires the integration of multiple sectors throughout the health system [4].

Following a disaster, nurses must meet the multiple care needs of large numbers of victims in a chaotic environment with limited resources. Disaster victims have a wide range of care needs that often exceed the available resources in the immediate aftermath of an event. For a better understanding of the mechanisms involved, it is necessary to develop comprehensive interpretations of the real operations within the response system that affect the disaster competencies demonstrated by nurses. The commands within the responding team are often guided by the stakeholders and administrators [19]. The views and descriptions gathered from administrators will reflect the real interpretations about how the competency standards are applied in practice. The initial phase of this study involves measuring the competency level. This is followed by discussion of the how the command system, through which roles and responsibilities are distributed during a disaster, understands the dynamic mechanisms within the responding team during a disaster event. To capture both sets of data, the study employs a mixed method approach, in which the investigator collects and analyses data, integrates the findings and draws inferences using both quantitative and qualitative approaches or methods in a single study or program of inquiry [16]. This research will be conducted in two phases. Phase one will involve quantitative measurement of disaster competencies among registered emergency nurses using a structured questionnaire. Phase Two will take the form of a qualitative study using interviews. This will be followed by an explanation of the circumstances leading to these outcomes in the context of the Saudi health system.

\section{Phase One: A Descriptive Quantitative Study}

According to Bowling (2014) [11] descriptive studies literally describe the phenomenon of interest and observed associations in order to estimate certain population parameters. This study will describe the level of disaster competency among emergency nurses in Saudi Arabia. Surveys are an effective tool for describing multiple dimensions of a study topic [16]. This study will use a questionnaire to determine the prevalence of disaster competency among nurses.

\section{Participants and Setting}

\section{Sampling Plan}

All registered nurses who are working in the emergency department in particular hospitals would be the accessible population for the study. These hospitals are King Fahad hospital with responsibility for responding to disaster in the north of the city, and King Abdul-Aziz hospital in the south, according to the Directorate of Health Affairs for disaster management in 


\section{Nursing \& Healthcare International Journal}

Jeddah. A Consecutive sampling involves recruiting all of the people from an accessible population who meet the eligibility criteria to form the sample of the study [11]. Although the representativeness of the data could be affected by such non-probability sampling, recruiting all emergency nurses within representative hospitals determined by Disaster Directorate which immediate calling in disaster as in consecutive sampling was considered the best approach for enhancing the representativeness of the sample as well as its generalizability to the target population [16].

To ensure the sample reflects a cross-section of the multiple degree levels held by emergency nurses, study participants must have either a Bachelor of Science in nursing or an Associate Degree in nursing, or have graduated from an accredited and approved nursing program. In addition, licensure is required, and may be obtained by graduating from an approved nursing program and passing the Saudi Health Speciality Examination.

\section{Controlling Confounders by Applying Explicit Inclusion Criteria}

Because nurses working in Saudi Arabia come from different countries, participants must have at least one year's experience in an emergency department in Saudi Arabia and at least one exposure to disaster response procedures to be included in the study. This sampling time must be sufficiently long to prevent potential biases [16]. In this study, requiring previous exposure to disaster response in Saudi Arabia or to a drill that enables participants to reflect the disaster competencies required under the Saudi health care system and thereby controls for unfamiliarity with the national disaster system.

As the sample includes participants with a range of educational backgrounds, the sample will be stratified based on whether they were educated in a Saudi Arabian nursing program or have some other educational background. This will both control confounder related to educational background and enhance the representativeness of the sample in relation to the target heterogenic population. This consideration involves stratification. Blocked in ensuring the study rigor and validity that enhance the representativeness of the sample in relation to the target heterogenic population, thereby ensuring the rigor and validity of the study [16].

The aim in recruiting all nurses assigned to work in emergency rooms is to produce a large sample size capable of ensuring representativeness and the statistical validity of any conclusion drawn from the study. More than 200 registered nurses work in the emergency rooms of two representative hospitals which are under the legislative authority of the Ministry of Health and which are on call as part of the disaster response strategy for Jeddah city.

\section{Data Collection}

The data collection tool in this phase of the study is a structured, standardized questionnaire which aims to measure knowledge and behaviour. Survey is a method of collecting data from a sample of the population of interest via a self-completed questionnaire. This is the novice survey has been used to demonstrate the disaster competency of nurses with the aim of developing robust standards of practice for application within the Saudi health care system.

A questionnaire was chosen as the data collection tool. The instrument will be uploaded on a specifically designed web page and distributed via email to the selected sample with a follow-up email from the administration office emphasizing the importance of this information to the research investigation. This will be followed five days by another email thanking those who have completed the questionnaire and encouraging any remaining participants to do so.

\section{Ethical Considerations}

Permission will be obtained in two phases. First ethical approval will be obtained from Ethics Committee of the Nursing and Health Sciences faculties at Queens University Belfast (QUB). Ethical approval from target hospitals will then be sought by contacting their Institutional Review Board committee at the Ministry of Health in Jeddah city. Informed consent signed attached with each questionnaire. To ensure the confidentiality of study participants and protect them from harmful procedures, all anonymous completed questionnaires will be discarded after the data has been transferred to the data analysis SPSS program, the findings are interpreted and the research has been approved for dissemination.

\section{Instrument}

The questionnaire developed by Althobaity, et al. (2016) [3] elicited quantitative data through different questioning styles. Validity and reliability are very important in quantitative studies to control measurement errors. Face and content validity were attained during the development of Althobaity, et al. (2016) [3] instrument. Their study undertook a principle component analysis by 


\section{Nursing \& Healthcare International Journal}

psychometrically testing the properties of each questionnaire items. A test-retest reliability ensured the same result each time of measurement. Additionally, Cronbach's' alpha coefficient was used with the goal of achieving the desirable 0.8 score, which signifies that items are highly interrelated [16].

The first section of the questionnaire asks for demographic data, for example, the respondent's age, nationality and gender. Questions4-13 ask for details of the respondent's highest nursing qualification, length of experience and any previous disaster training. This group of questions also seeks to distinguish between those respondents who trained as nurses in Saudi Arabia and those who trained in any country other than Saudi Arabia, and whether the respondent has undertaken any other form of disaster training, whether in or outside the hospital. The last question in this section asks whether the respondent has been actively involved in a real disaster response incident in the last ten years.

The second section uses a Likert scale to explore the respondent's role and responsibilities in disaster response. Possible responses ranges from Never to Most Often have been in their role and responsibilities. This section reflects the role of nurses at various levels in the hierarchy of authority. At the top of this hierarchy, are those who are actively involved in disaster decision and policy making. At the next level are those who are actively engaged in planning the response to disaster. These are followed by those who take part in ongoing training drills in the workplace.

The questions in the third section are designed to assess the importance of each of 30 disaster competencies extracted from the ICN's Framework for Disaster Nursing Competencies, which apply to all disaster management phases. The fourth section explores the challenging factors that interfere with disaster nursing in Saudi Arabia.

The questionnaire will be distributed by email to the participants. Hospital staff have secure access to an internal system and Committee number as member in Saudi Commission for Health Speciality to access that system. A consent form will be provided as the cover page to the questionnaire. The participant's signature will indicate their willingness to be part of the study.

\section{Data Analysis}

Data will be statistically analysed using the software program SPSS. SPSS Statistics is a software package used for logical statistical analysis. When using this program, the variables will be defined in the data view column.

This process will involve the following: labeling to distinguish variables in the label column; inserting all obtained data within the program table cells with their values; completing all data records with a view to preserving all options for analysis; and importing the data to a software spreadsheet for display.

For a deeper understanding of the factors that both enhancing and hinder nurses' preparedness for disaster management, individual interviews are planned with supervisors and directors in the second phase of the study.

\section{Phase Two: Qualitative Study}

In Phase Two of the study, individual interviews will be conducted with policy makers and supervisors to obtain their view of the current state of disaster competency in disaster field resilient health care systems in Saudi Arabia.

Although interviews are time consuming, the information they generate enables broader explanations and more detailed discussion of the factors affecting a particular area [11]. A deeper understanding of disaster nurses' performance in the disaster field can be obtained through interviews with higher authorities. Because the aim of this phase of the study understands the mechanisms guiding the standards for assessing the response to disasters, gathering comprehensive details from response managers will enable social interpretations within this context.

\section{Participants}

A purposive sample will be invited to make a voluntary contribution to this study. Because of the multilevel relationship between stakeholders, this will involve selecting samples from different levels of the hierarchy [16].

An invitation letter will be delivered by mail or electronic email to administrators and stakeholders granted authority by the Saudi Health Speciality and who have authority to manipulate workforces in case of a disaster. The researcher will begin with volunteer participants and then pursue continuous recruiting through snowballing until data saturation is achieved [16].

Each interview will take place inside the supervisor's 


\section{Nursing \& Healthcare International Journal}

office and will last roughly one hour, during which the participant's thoughts about the current state of disaster nursing in Saudi Arabia will be explored. The interview will be tape recorded. Permission to record the interview will be obtained before the interview begins Arrangements for transcribing these recordings will be addressed in the application to be submitted to the Ethics Committee.

\section{Data Collection}

Data will be collected through semi-structured interviews to obtain a deeper understanding of dynamic mechanisms that inform the work of the disaster response authorities and incident commanders'. Open- ended questions will raise issues beyond disaster response standards within the Saudi health care system.

\section{Interview Protocol}

Questions will evolve from the disaster response framework applied within the Saudi health care system. Saudi Arabia is one of 186 countries that have made a commitment to achieving a resilient health care system. Preparing nurses to be competent disaster responders is one of the priorities of the Disaster Risk Reduction Framework. The ability of disaster teams to demonstrate disaster competencies is a crucial element in resilient health care [19-21]. Therefore, the discussion will focus on the stakeholder's view and assessment standards for nurses' performance in a disaster field.

Each interview will begin by introducing the researcher's identity and qualifications (e.g., a licensed member of the Saudi Health Speciality Committee) in order to establish a trusting and relaxed environment. One option being considered is to open the dialogue with...opening the dialogue with a scenario of a real disaster event and a role play of the disaster response to solicit the participant's interpretation of the nurses' performance.

The researcher will prepare a list of areas and topics to be covered within the interview. These prompt questions are used to guide the topics and the participants are free to elaborate and explain as much as they choose [11]. In relation to Research Question No. 4, participants will be encouraged to address the Incident Command Systems used in the healthcare institutions and their mechanisms. Other topics to explore include interprofessional relations and interactions; operations within resilient response systems in Saudi Arabia; all possible circumstances surrounding the disaster nursing response; and the best approaches for applying disaster standards in real disaster fields.

\section{Data Analysis}

All data extracted from transcripts will be coded and thematic categorizing in preparation for analysis [11]. Data person triangulation involves collecting data from different levels of people, for example, in this study, from emergency nurses and their stakeholders, with the aim of validating the data from multiple perspectives on the research topic. To achieve "contextualization" of the research topic is the main reason for triangulation of the data. This will lead to generalizability to broader concepts and transferability of the findings, which can then be applied to different study groups [16].

\section{Data Validation}

All transcripts will be generated by the software program that transfers the participants' speech into a written format in order to obtain high-quality transcripts.

Reviewing one's own interviewing style can enhance the quality of future interviews [11]. In addition, all transcripts will be reviewed with supervisors with the aim of reaching agreement regarding the participant's intent.

Furthermore, the participants will be invited to review the researcher's interpretations of their transcripts to ensure the credibility of the data collected. The written feedback comments obtained from this member checking will provide additional rigor. By reviewing the transcripts and the researchers' interpretations it will be possible to determine the speaker's intended meaning and actual explanations in written feedback comments on the researcher's interpretation.

\section{Integrations and Inferences in Mixed Method}

For the purpose of the convergent mixed method design of this study, both quantitative and qualitative data will be collected simultaneously.

To facilitate the display of data and in order to extract patterns and make comparisons with diverse information, constructing Meta-Matrices is a widely used approach involving the use of matrices. In addition, the themes obtained from the interviews transcripts will be summarized. In this study, the competency determinations will be integrated with all the mechanisms within the disaster response team within the disaster 


\section{Nursing \& Healthcare International Journal}

response healthcare standards, making it possible to describe the relationship between the disaster nurses performance in the context of disaster resilient healthcare.

\section{Dissemination}

The research proposal was presented at the international Disaster Risk Reduction conference in Davos, Switzerland, 2016 as an outstanding study on the United Nations Disaster Standards from the perspective of nursing preparedness. The results from this evaluation of current disaster competencies against the standards developed by ICN will be submitted to a high-impact journal for publication, as will the analysis of the data collected from Saudi health care stakeholders regarding the factors which prevent Saudi nurses from developing disaster preparedness in accordance with the United Nation disaster preparedness goal of resilient health care. Opportunities for further research will be identified and recommendations made for transferring the learning from this study to the other 185 countries which have signed up to the United Nations Disaster Reduction Framework.

\section{References}

1. CRED (2015) The Human Cost of Weather Related Disasters, 1995-2015. United Nations Office for Disaster Risk Reduction, Centre for Research on the Epidemiology of Disasters, pp: 30.

2. WHO \& ICN (2009) ICN Framework of Disaster Nursing Competencies, World Health Organization \&International Council of Nurses, Geneva: ICN, pp: 84.

3. Althobaity A, Plummer V, Innes K, Copnell B (2015) Perceptions of knowledge of disaster management among military and civilian nurses in Saudi Arabia. Australas Emerg Nurs J 18(3): 156-164.

4. Abosuliman SS, Kumar A, Alam F (2013) Disaster preparedness and management in Saudi Arabia: An empirical investigation. International Journal of Economics and Management Engineering 7(12): 3256-3260.

5. Kirsch T, Sauer L, Guha Sapir D (2012) Analysis of the international and US response to the Haiti earthquake: Recommendations for change. Disaster Medicine Public Health Preparedness 6(3): 200-208.

6. Anderson DA (2012) Exploration of Nurse and Nurse Leaders' Reports of the Competencies Needed by
Rural Registered Nurses in Disasters, University of Hartford.

7. Cusack L, Gebbie K (2015) Call for national dialogue: Adapting standards of care in extreme events not ready. Collegian 24(1): 93-100.

8. Daily E, Padjen P, Birnbaum M (2010) A review of competencies developed for disaster healthcare providers: Limitations of current process and applicability. Prehosp Disaster Med 25(5): 387-395.

9. Schultz CH, Koenig KL, Whiteside M, Murray R (2012) Development of national standardized all-hazard disaster core competencies for acute care physicians, nurses, and EMS professionals. Annuals of Emergency Medicine 59(3): 196-208.

10. UNISDR (2015) Sendai Framework for Disaster Risk Reduction 2015-2030, United Nations Office for Disaster Risk Reduction, pp: 1-37.

11. Bowling A (2014) Research Methods in Health: Investigating Health and Health Services. New York, NY: Open University Press.

12. Alraga SM (2017) An investigation into disaster health management in Saudi Arabia. Journal of Hospital \& Medical Management 3(2): 18.

13. UNISDR (2018) The global platform for disaster risk reduction, United Nation International Strategy for Disaster Reduction.

14. Bartlett HP, Simonite V, Westcott E, Taylor HR (2000) A comparison of the nursing competence of graduates and diplomates from UK nursing programmes. J ClinNurs 9(3): 369-379.

15. Cresswell LJW (2017) Research design: Qualitative, quantitative, and mixed methods approach California: Sage Publications.

16. Polit D, Beck C (2017) Nursing Research: Generating and Assessing Evidence for Nursing Practice $10^{\text {th }}$ (Edn.), Philadelphia, PA: Wolters Kluwer.

17. Howarth E, Devers K, Moore G, O Cathain A, Dixon Woods M (2016) Contextual issues and qualitative research. In: Raine $\mathrm{R}$, et al. (Eds), Challenges, solutions and future directions in the evaluation of service innovations in health care and public health. Health Services and Delivery Research, Essay 7. Southampton (UK): NIHR Journals Library 4(16). 
18. Al Thobaity A, Williams B, Plummer V (2016) A new scale for disaster nursing core competencies: Development and psychometric testing. Australas Emerg Nurs J 19(1): 11-19.

19. Drenkard K, Rigotti G, Hanfling D, Fahlgren TL, La Francois G (2002) Healthcare system disaster preparedness, part 1: Readiness planning. J Nurs Adm 32(9): 461-469.
20. Alfred D, Chilton J, Connor D, Deal B, Fountain R, et al. (2015) Preparing for disasters: Education and management strategies explored. Disaster Management Nursing Education 15(1): 82-89.

21. BBC website noted that Some Saudis took to social media to complain of what they said was inadequate infrastructure in the area.

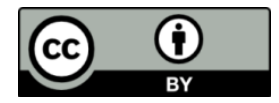

